\title{
Vandetanib for the Management of Advanced Medullary Thyroid Cancer: A Real-World Multicenter Experience
}

\author{
Mijin Kim ${ }^{1, *}$, Jee Hee Yoon ${ }^{2 *}$, Jonghwa Ahn³, Min Ji Jeon ${ }^{3}$, Hee Kyung Kim², Dong Jun Lim4, Ho-Cheol Kang², \\ In Joo Kim ${ }^{1}$, Young Kee Shong ${ }^{3}$, Tae Yong Kim ${ }^{3}$, Bo Hyun Kim ${ }^{1}$ \\ ${ }^{1}$ Department of Internal Medicine, Biomedical Research Institute, Pusan National University Hospital, Busan; ${ }^{2}$ Department of \\ Internal Medicine, Chonnam National University Hwasun Hospital, Hwasun; ${ }^{3}$ Department of Internal Medicine, Asan Medical \\ Center, University of Ulsan College of Medicine; ${ }^{4}$ Department of Internal Medicine, Seoul St. Mary's Hospital, College of \\ Medicine, The Catholic University of Korea, Seoul, Korea
}

Background: Vandetanib is the most widely used tyrosine kinase inhibitor for the treatment of patients with advanced medullary thyroid cancer (MTC). However, only limited data regarding its use outside clinical trials are available. We aimed to evaluate the efficacy and safety of vandetanib in patients with advanced MTC in routine clinical practice.

Methods: In this multicenter retrospective study, 12 patients with locally advanced or metastatic MTC treated with vandetanib at four tertiary hospitals were included. The primary outcome was the objective response rate (ORR) based on the Response Evaluation Criteria in Solid Tumors. The progression-free survival (PFS), overall survival (OS), and toxicities were also evaluated.

Results: Eleven patients (92\%) had distant metastasis and $10(83 \%)$ had disease progression at enrollment. Partial response was observed in five patients (ORR, 42\%) and stable disease lasting $\geq 24$ weeks was reported in an additional five patients (83\%). During the median 31.7 months of follow-up, disease progression was seen in five patients (42\%); of these, two died due to disease progression. The median PFS was 25.9 months, while the median OS was not reached. All patients experienced adverse events (AEs) which were generally consistent with the known safety profile of vandetanib. Vandetanib was discontinued in two patients due to skin toxicity.

Conclusion: Consistent with the phase III trial, this study confirmed the efficacy of vandetanib for advanced MTC in terms of both ORR and PFS in the real-world setting. Vandetanib was well tolerated in the majority of patients, and there were no fatal AEs.

Keywords: Thyroid neoplasms; Protein kinase inhibitors; Progression-free survival; Toxicity

Received: 21 April 2020, Revised: 23 June 2020, Accepted: 16 July 2020 Corresponding authors: Bo Hyun Kim

Division of Endocrinology and Metabolism, Department of Internal Medicine, Biomedical Research Institute, Pusan National University Hospital, 179 Gudeokro, Seo-gu, Busan 49241, Korea

Tel: +82-51-240-7236, Fax: +82-51-254-3237, E-mail: pons71@hanmail.net

Tae Yong Kim

Division of Endocrinology and Metabolism, Department of Internal Medicine, Asan Medical Center, University of Ulsan College of Medicine, 88 Olympic-ro 43-gil, Songpa-gu, Seoul 05505, Korea

Tel: +82-2-3010-3249, Fax: +82-2-3010-6962, E-mail: tykim@amc.seoul.kr

\section{Copyright $\odot 2020$ Korean Endocrine Society}

This is an Open Access article distributed under the terms of the Creative Commons Attribution Non-Commercial License (https://creativecommons.org/ licenses/by-nc/4.0/) which permits unrestricted non-commercial use, distribution, and reproduction in any medium, provided the original work is properly cited.

*These authors contributed equally to this work. 


\section{INTRODUCTION}

Medullary thyroid cancer (MTC) originates from the parafollicular $\mathrm{C}$ cells of the thyroid gland and represents $5 \%$ of all thyroid cancers [1]. MTC is a relatively indolent tumor, and the 10-year overall survival (OS) rate is approximately $75 \%$ to $80 \%$ [2]. However, patients with unresectable, locally advanced or metastatic MTC have limited therapeutic options, and their 10-year OS rate is reported to be $40 \%$ or less [2]. Owing to limited responsiveness to non-surgical treatments (radioactive iodine therapy, external radiotherapy, and conventional chemotherapy), targeted therapy with tyrosine kinase inhibitors (TKIs) has emerged as an important therapeutic option for patients with advanced MTC [3-6].

Vandetanib is the most widely used TKI for advanced MTC, targeting the RET oncogene, vascular endothelial growth factor receptor (VEGFR), and epidermal growth factor receptor (EGFR) [6]. Based on the encouraging results of the international, multicenter, randomized phase III clinical trial (ZETA trial), vandetanib was approved by the Food and Drug Administration and the European Medical Agency for the treatment of patients with symptomatic, unresectable, locally advanced or metastatic MTC [7]. In the ZETA trial, vandetanib showed a significant prolongation of progression-free survival (PFS) and a high objective response rate (ORR) compared with placebo [7]. Although the ZETA trial showed the efficacy and safety of vandetanib, only limited data regarding its use outside clinical trials are available $[7,8]$. In this multicenter cohort study, we aimed to evaluate the efficacy and safety of vandetanib in patients with locally advanced or metastatic MTC, when vandetanib was administered in routine clinical practice.

\section{METHODS}

\section{Patients and study design}

In this multicenter, retrospective cohort study, 12 patients with advanced MTC who were treated with vandetanib from May 2015 to December 2019 at four tertiary hospitals in Korea were included. Eligible patients were aged $>18$ years and had measurable, unresectable, locally advanced or metastatic MTC. Patients who were not assessable for the objective tumor response rate by the Response Evaluation Criteria in Solid Tumors (RECIST) or who were deemed candidates for local treatment modalities were excluded. All patients were pathologically confirmed to have MTC and were treated with vandetanib at a starting dose of $300 \mathrm{mg}$ daily. This study protocol was approved by all the relevant institutional ethical committees (Asan Medical Center, 2019-1399; Chonnam National University Hwasun Hospital, CNUHH-2020-051; Pusan National University Hospital, 1910-010-083; and Seoul St. Mary's Hospital, KC20RIDI0362). Written informed consent by the patients was waived due to a retrospective nature of our study.

\section{Evaluation of efficacy}

Tumor responses were evaluated based on investigator assessment at 2 to 3 -month intervals during treatment. The primary objective of this study was to assess the ORR with vandetanib, which was defined as the proportion of patients who show complete response (CR) or partial response (PR) as the best response, according to RECIST, version 1.1 [9]. Additionally, we assessed the disease control rate (DCR), biochemical response rate, PFS, and OS. DCR was defined as the percentage of patients who achieved CR, PR, or stable disease (SD) for $\geq 24$ weeks. Biochemical response was determined by changes in serum levels of calcitonin and carcinoembryonic antigen (CEA) and was defined as follows: $\mathrm{CR}$, complete normalization of serum levels after treatment; $\mathrm{PR}, \geq 50 \%$ decrease from baseline, maintained for a minimum of 4 weeks; SD, $<50 \%$ decrease or $\geq 20 \%$ increase from baseline, maintained for at least 4 weeks; or progressive disease, $>20 \%$ increase from baseline, maintained for at least 4 weeks [10]. PFS was defined as the time from vandetanib administration to the date of the first documentation of disease progression according to RECIST version 1.1, and OS was defined as the time from the first dose of vandetanib until death from any cause.

\section{Safety and tolerability}

Safety and tolerability were assessed throughout the administration of vandetanib. For safety assessment, adverse events (AEs); vital signs; results of clinical chemistry, hematology, and urinalysis; electrocardiograms (ECGs); and Eastern Cooperative Oncology Group (ECOG) performance status were monitored at baseline and at every visit during follow-up. AEs were graded based on the National Cancer Institute Common Terminology Criteria for Adverse Events, version 4.0. If a causal relation between AEs and vandetanib could not be ruled out, events were classified as treatment-related AEs.

\section{Statistical analysis}

Statistical analysis was performed using the R program version 3.5.1 (R Foundation for Statistical Computing, Vienna, Austria; http://www.R-project.org). Continuous variables were presented 
as medians with interquartile ranges (IQRs) and categorical variables were presented as numbers with percentages. Percent changes in the total sum of target lesion diameters were calculated and PFS and OS curves were drawn using Graph Pad Prism version 5.0 (GraphPad Software, SanDiego, CA, USA; http://www.graphpad.com). Estimates of median PFS and OS were obtained using the Kaplan-Meier method.

\section{RESULTS}

\section{Patients}

Table 1 summarizes the demographics and characteristics of 12

Table 1. Baseline Characteristics

\begin{tabular}{lc}
\hline Characteristic & Value \\
\hline Age, yr & $59.0(54-64)$ \\
Male sex & $6(50)$ \\
Disease type & \\
Locally advanced & $1(8)$ \\
Metastatic & $11(92)$ \\
Liver & $7(58)$ \\
Lung & $8(67)$ \\
Bone & $4(33)$ \\
Lymph nodes & $9(75)$ \\
Neck & $1(8)$ \\
Brain & $3(25)$ \\
Pancreas & $1(8)$
\end{tabular}

Prior treatment

Thyroidectomy $12(100)$

Re-surgery $5(36)$

Radioactive iodine therapy $1(8)$

Radiation therapy 4 (33)

Systemic chemotherapy $2(17$; sorafenib)

RET mutation

Positive $1(8)$

Negative $\quad 8(67)$

Unknown $3(25)$

Target lesions

Liver $\quad 7(58)$

Lung $4(33)$

Lymph nodes $3(25)$

Neck $1(8)$

Pancreas $1(8)$

Progressive disease at vandetanib initiation $\quad 10(83)$

Symptoms at vandetanib initiation $3(25)$

Values are expressed as median (interquartile range) or number (\%). patients with advanced MTC who received vandetanib between May 1, 2015 and December 31, 2019. Median age at the initiation of vandetanib treatment was 59.0 years (IQR, 54.4 to 64.2), and six patients $(50 \%)$ were male. Most patients $(n=11,92 \%)$ had distant metastasis, most frequently to the lungs $(67 \%)$ and liver (58\%). Before vandetanib administration, all patients had undergone thyroidectomy, seven (58\%) had undergone metastasectomy, two (17\%) had received sorafenib, and four (33\%) had received external radiotherapy. One patient had both MTC and papillary thyroid cancer and had received prior radioactive iodine therapy. RET genetic screening was performed in nine patients $(75 \%)$, and the majority of patients $(8 / 9,89 \%)$ were not carrying a RET mutation. A germline mutation in the RET gene (p.D878Y) was identified in one patient. At the time of vandetanib treatment initiation, 10 patients $(83 \%)$ had progressive disease and three patients (25\%) showed cancer-related symptoms. At the data cutoff (December 31, 2019), five patients (42\%) were receiving treatment, whereas vandetanib had been discontinued in five (42\%) and two (17\%) patients because of disease progression and AEs, respectively.

\section{Efficacy}

Median duration of vandetanib treatment was 25.3 months (range, 2.5 to 49.3), and median follow-up duration since vandetanib initiation was 31.7 months (range, 13.1 to 49.3). Tumor burden according to the sum of the largest diameter of target lesions decreased in 10 patients (83\%); median of the best percent change was $-24.1 \%$ (Fig. 1). All patients had an evaluable radiological tumor response; five (42\%) and seven (58\%) patients exhibited PR and SD, respectively (Table 2, Fig. 1). Among the patients who achieved SD, five had a durable response for 24 weeks or longer (Table 2). Overall, the estimated ORR was $42 \%$, and the DCR was $83 \%$. During vandetanib administration, we also observed reductions in calcitonin and CEA levels. Serum calcitonin and CEA levels decreased by $50 \%$ or more in eight patients (67\%). The median PFS was 25.9 months (Fig. 2A), and the median OS was not reached at the time of data cutoff (Fig. 2B). At the end of the follow-up, five patients (42\%) developed progressive disease after a median of 17.1 months (range, 6.7 to 25.9) from vandetanib administration. Among them, three patients showed disease progression in target lesions, one showed disease progression in the form of a non-target lesion in the target organ (liver), and one showed a new metastatic lesion (bone) without progression in the target lesion. The latter two patients with cancer progression in non-target lesions died due to disease progression. 
Table 2. Best Response to Vandetanib in Patients with Medullary Thyroid Cancer

\begin{tabular}{|c|c|}
\hline Disease response & No. $(\%)$ \\
\hline Complete response & 0 \\
\hline Partial response & $5(42)$ \\
\hline Stable disease & $7(58)$ \\
\hline Progressive disease & 0 \\
\hline Objective response rate & $5(42)$ \\
\hline Disease control rate & $10(83)$ \\
\hline Biochemical response rate: calcitonin & $8(67)$ \\
\hline Biochemical response rate: CEA & $8(67)$ \\
\hline \multicolumn{2}{|c|}{$\begin{array}{l}\text { Tumor response was assessed using the Response Criteria in Solid Tu- } \\
\text { mors, version } 1.1 \text {. The objective response rate was calculated as com- } \\
\text { plete response (CR) plus partial response (PR). The disease control rate } \\
\text { was calculated as CR plus PR plus stable disease } \geq 24 \text { weeks. The bio- } \\
\text { chemical response was defined by decreases of } 50 \% \text { or more in serum } \\
\text { calcitonin and CEA levels which were maintained for at least } 4 \text { weeks. } \\
\text { CEA, carcinoembryonic antigen. }\end{array}$} \\
\hline
\end{tabular}

\section{Safety and adverse effects}

All patients experienced AEs; the common AEs (of any grade) and grade 3 or higher AEs observed during the treatment are summarized in Table 3. With a median dose of $200 \mathrm{mg} /$ day, vandetanib dose reduction, interruption, and discontinuation were required in four (33\%), six (50\%), and two (17\%) patients, respectively. The frequent AEs that occurred in more than 50\% of patients receiving vandetanib were diarrhea $(92 \%)$, rash (83\%), hypothyroidism (75\%), decreased appetite (67\%), and fatigue $(67 \%)$. mucositis $(50 \%)$, corrected QT (QTc) prolongation (50\%), palmar-plantar erythrodysesthesia syndrome (42\%), and abdominal pain $(42 \%)$ were also common AEs. Grade 3 or 4 AEs included rash (33\%), diarrhea (25\%), mucositis (17\%), QTc prolongation (17\%), decreased appetite (8\%), palmar-plantar erythrodysesthesia syndrome (8\%), abdominal pain (8\%), and blurred vision (8\%). The baseline QTc interval prior to vandetanib administration in all patients was $<450 \mathrm{~ms}$. QTc prolon-

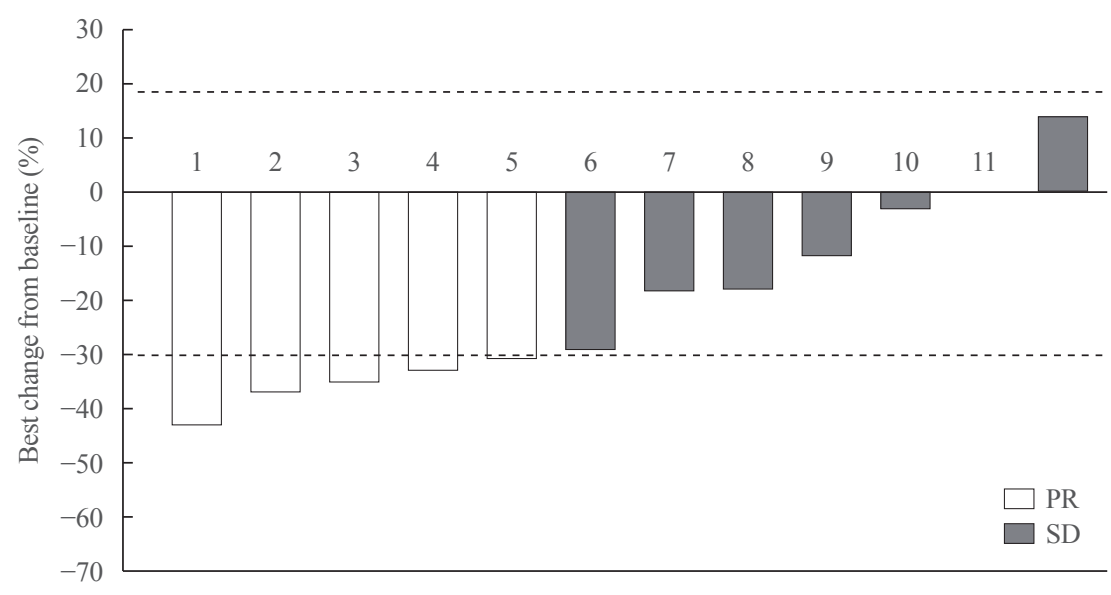

Fig. 1. Best percent change in the total sum of target lesion diameters. PR, partial response; SD, stable disease.
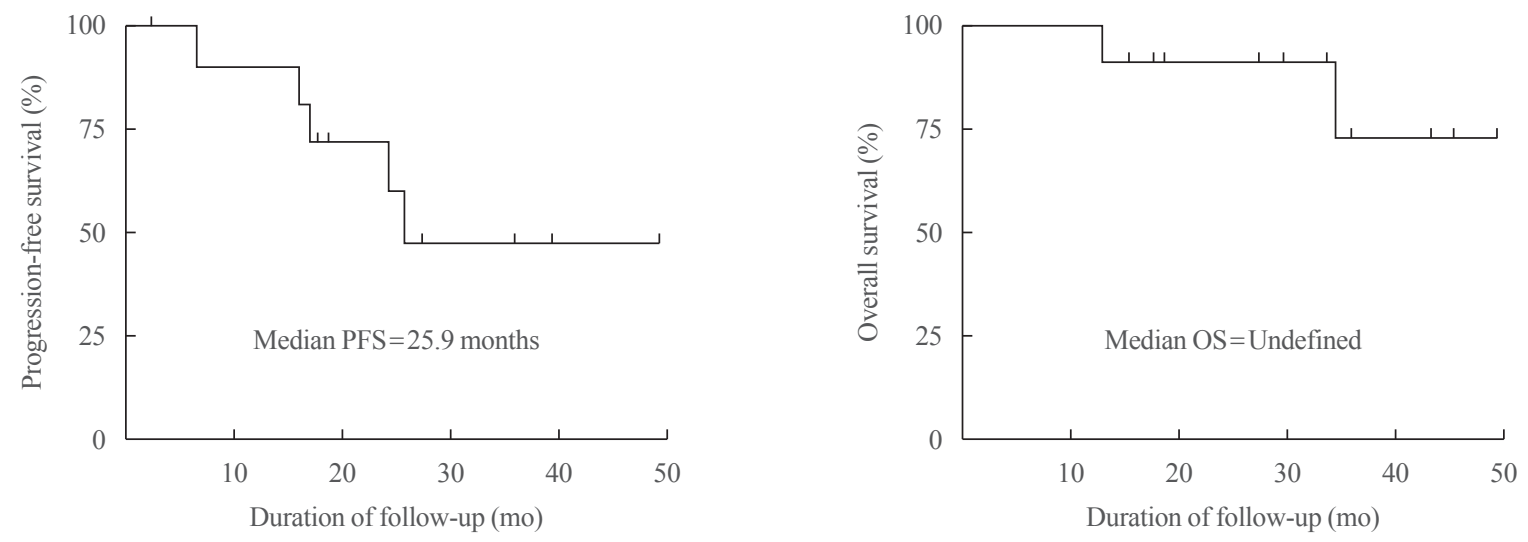

No. at risk $12 \quad 10 \quad 6 \quad 3$

0

A No. at risk 12 12 8

6

Fig. 2. Kaplan-Meier plots for (A) progression-free survival (PFS) and (B) overall survival (OS). 
Table 3. Adverse Events Occurring in Patients with Medullary Thyroid Cancer Treated with Vandetanib

\begin{tabular}{lcc}
\hline Adverse events & $\begin{array}{c}\text { Any } \\
\text { grade }\end{array}$ & $\begin{array}{c}\text { Grade } \\
3-4\end{array}$ \\
\hline Treatment-related adverse effects & $12(100)$ & $6(50)$ \\
Diarrhea & $11(92)$ & $3(25)$ \\
Rash & $10(83)$ & $4(33)$ \\
Hypothyroidism & $9(75)$ & 0 \\
Decreased appetite & $8(67)$ & $1(8)$ \\
Fatigue & $8(67)$ & 0 \\
Mucositis & $6(50)$ & $2(17)$ \\
QTc prolongation & $6(50)$ & $2(17)$ \\
Palmar-plantar erythrodysesthesia syndrome & $5(42)$ & $1(8)$ \\
Abdominal pain & $5(42)$ & $1(8)$ \\
Nausea & $2(17)$ & 0 \\
Hypertension & $2(17)$ & 0 \\
Acne or folliculitis & $2(17)$ & 0 \\
Blurred vision & $1(8)$ & $1(8)$ \\
Headache & $1(8)$ & 0 \\
Alopecia & $1(8)$ & 0 \\
Hypocalcemia & $1(8)$ & 0 \\
Gum bleeding & $1(8)$ & 0 \\
\hline
\end{tabular}

Values are expressed as number (\%). Adverse events were classified based on the National Cancer Institute Common Terminology Criteria for Adverse Events, version 4.0.

QTc, corrected QT.

gation was not related to changes in plasma calcium levels, but the need for calcium and vitamin D replacement increased in one patient with established hypoparathyroidism. Skin toxicity led to treatment discontinuation in two patients; there were no fatal AEs.

\section{DISCUSSION}

This study evaluated the efficacy and toxicity of vandetanib in routine practice in patients with locally advanced or metastatic MTC, during a median follow-up of 32 months. Almost all patients $(92 \%)$ had distant metastasis and $83 \%$ of patients had progressive disease at vandetanib initiation. The ORR and DCR were $42 \%$ and $83 \%$, respectively, and these values correspond with the results of the ZETA trial (ORR 45\% and DCR 87\%) [7]. The median PFS was 25.9 months, which was slightly shorter than that of patients treated with vandetanib (30.5 months) while longer than that of patients treated with placebo
(19.3 months) in the ZETA trial [7]. With a median dose of 200 $\mathrm{mg} /$ day, vandetanib was discontinued in two patients due to skin toxicity. The AEs were generally consistent with the known safety profile of vandetanib, and most were manageable by dose modification and medical therapy.

Vandetanib is the first approved TKI for advanced MTC treatment that selectively targets $R E T$, VEGFR, and EGFR [6,11]. It was approved based on the results of phase II and III trials, which demonstrated a significant durable response and prolongation of PFS $[7,10,12]$. Thereafter, data from retrospective studies have supported the positive clinical impact of vandetanib $[8,13]$. A retrospective study analyzing 60 MTC patients receiving vandetanib outside any trial reported an ORR of $22 \%$ with a median PFS of 16.1 months [8]. In another retrospective study of 11 patients with progressive metastatic MTC, the rate of PR was $36 \%$ and that of SD for 6 months or longer was $27 \%$, but the median PFS was not reached during the median treatment duration of 7 months [13]. Recent studies have reported prognostic indicators predicting better and longer response to vandetanib in advanced MTC patients [14-17]. More advantages of vandetanib therapy have been reported in MTC patients who are younger, have a good ECOG performance status, and have symptomatic disease [17]. Pre-therapeutic fluorine-18-fluorodeoxyglucose positron emission tomography/computed tomography also provides prognostic information in patients with advanced and progressive MTC [16]. However, the presence of RET mutations is not a predictor of treatment efficacy of vandetanib, consistent with the results previously shown in clinical trials $[7,10,17]$. Selective and highly active $R E T$ inhibitors which are currently under clinical trial may provide a promising treatment option for MTC patients with RET mutations [18]. Due to the limited number of patients in the current study, we could not identify the clinical features associated with better clinical outcomes after vandetanib treatment.

This study confirms the efficacy of vandetanib in patients with advanced MTC, as shown in the ZETA trial, outside of a clinical trial [7]. The study population differed between our study and the ZETA trial. Patients enrolled in the ZETA trial were required to have measurable disease but not progressive disease at enrollment [7]. This inclusion criterium may have allowed the enrollment of many patients with indolent disease in the ZETA trial, as evidenced by a PFS of 19.3 months in the placebo group [7]. In contrast, the majority of patients (83\%) included in our study had progressive disease at the time of vandetanib initiation. The inclusion criteria in this study also did not include progressive disease at enrollment, however, these 
patients had disease progression, according to RECIST, within 6 months (range, 1 to 6 ) before vandetanib administration. Another difference was the proportion of MTCs with RET mutations. The association between RET mutation and vandetanib efficacy was inconclusive in previous studies [7,10,17], but MTC patients harboring the M918T mutation showed more aggressive disease and better response rates to vandetanib therapy than those without the RET918 mutation [15]. The proportion of patients with cancers harboring a RET mutation was $8 \%$ in our study, while it was $59 \%$ in the treatment group of the ZETA trial. Furthermore, in our study, patients previously treated with TKIs were allowed to participate, whereas the ZETA trial did not report data on previous TKI therapy. Despite these differences, the results of our study and the ZETA trial were similar with respect to the ORR and PFS prolongation.

Although the frequency of AEs in our study was higher than that observed in the ZETA trial, no unexpected AEs occurred during treatment. Additionally, most AEs were mild and manageable by dose modification and medical therapies. Dose reduction, interruption, and discontinuation was needed in $33 \%$, $50 \%$, and $17 \%$ of patients, respectively. Consistent with the findings of the ZETA trial, the most common AEs were diarrhea, rash, hypothyroidism, decreased appetite, fatigue, and QTc prolongation [7]. It is significant that the number of patients who required an increase in the daily dose of levothyroxine (75\% vs. $49 \%$ ) and who developed QTc prolongation (50\% vs. 14\%) was higher in our study than in the ZETA trial. The reason this study has a higher frequency of QTc prolongation than the ZETA trial was because EGC was monitored more frequently and the definition of QTc prolongation was different. In the ZETA trial, scheduled ECGs were performed during screening, at 1, 2, 4, 8, and 12 weeks and every 3 months thereafter [7]. Meanwhile, in this study, ECGs were monitored at baseline and evaluated at 1 to 2-week intervals for a month, then 1 to 2-month intervals. QTc prolongation was recorded according to the Common Terminology Criteria for Adverse Events (CTCAE) version 4 in our study: grade 1, QTc interval 450 to $480 \mathrm{~ms}$; grade 2, 480 to $500 \mathrm{~ms}$; grade $3, \geq 501 \mathrm{~ms}$ on at least two separate ECGs; and grade 4, $\geq 501$ or $>60 \mathrm{~ms}$ change from baseline and torsade de pointes or polymorphic ventricular tachycardia or signs/symptoms of serious arrhythmia. In the ZETA trial, QTc prolongation was defined as a single measurement $\geq 550 \mathrm{~ms}$ or an increase of $\geq 100 \mathrm{~ms}$ from baseline, two consecutive measurements (within 48 hours of each other) that were $\geq 500$ but $<550 \mathrm{~ms}$, or an increase of $\geq 60$ but $<100 \mathrm{~ms}$ from baseline to a value $\geq 480 \mathrm{~ms}$ [7]. Therefore, the ZETA trial that used this criteria to identify QTc pro- longation may have missed some events since patients whose QTc interval ranged from 450 to $480 \mathrm{~ms}$ were not recorded. This evidence suggests that thyroid function, ECGs, and electrolyte levels should be monitored frequently after vandetanib initiation, to allow for early management of AEs before they worsen. It is also noteworthy that cutaneous AEs led to drug discontinuation in two patients in our study. The etiology of rash induced by vandetanib is unclear, but it is likely to be associated with the inhibition of EGFR [19-21]. Reduction of quality of life due to rash may play a significant role in adherence to the drug. Therefore, awareness and treatment of cutaneous AEs is needed to improve drug compliance and to maximize the dose while ensuring the best clinical benefits.

Because patients with advanced MTC can present with indolent disease and long life expectancy, the median OS was not reached during the median follow-up of 32 months. Previous data on the ability of vandetanib to improve survival are limited $[7,10,12,17,22]$. OS data were also immature at the data cutoff in the ZETA trial, and an interim analysis showed no significant survival benefits with vandetanib treatment, during a median duration of 24 months of the randomized phase [7]. These results demonstrate that once vandetanib has been started, the patient is likely to receive this drug for a long time. In addition to doubtful survival benefits and toxicities caused by the chronic use of vandetanib, it is necessary to consider the escape phenomenon and rapid tumor growth after drug withdrawal while determining vandetanib initiation $[6,22,23]$. Therefore, it is crucial to establish an appropriate risk stratification based on patients with advanced MTC who have benefited with vandetanib administration, and only a selected group of patients should be considered for vandetanib therapy.

The limitations of our study are mainly related to its retrospective nature and the small number of patients. The treatment duration was longer than that in the ZETA trial, but it was relatively short to evaluate the median OS. Further, information on RET mutational status was not available in $25 \%$ of patients. Despite these limitations, our study is the first in Korea to evaluate the efficacy and safety of vandetanib in patients with advanced MTC in a real-world setting.

This multicenter, real-world analysis supports the results of the ZETA trial in that vandetanib has clinical benefits with regards to ORR and PFS in patients with advanced MTC. Although all patients experienced any-grade AEs, there were no fatal AEs; all AEs were generally consistent with the known safety profile of vandetanib. In conclusion, vandetanib is an effective treatment option in routine clinical practice with signifi- 
cant efficacy and manageable safety for advanced MTC.

\section{CONFLICTS OF INTEREST}

No potential conflict of interest relevant to this article was reported.

\section{AUTHOR CONTRIBUTIONS}

Conception or design: M.K., M.J.J., D.J.L., H.C.K., Y.K.S., T.Y.K., B.H.K. Acquisition, analysis, or interpretation of data: M.K., J.H.Y., J.A., M.J.J., H.K.K., D.J.L., H.C.K., I.J.K., Y.K.S., T.Y.K., B.H.K. Drafting the work or revising: M.K., J.H.Y., M.J.J., H.K.K., D.J.L., H.C.K., I.J.K., Y.K.S., T.Y.K., B.H.K. Final approval of the manuscript: M.K., J.H.Y., J.A., M.J.J., H.K.K., D.J.L., H.C.K., I.J.K., Y.K.S., T.Y.K., B.H.K.

\section{ORCID}

Mijin Kim https://orcid.org/0000-0002-1538-8859

Jee Hee Yoon https://orcid.org/0000-0002-5919-6162

Tae Yong Kim https://orcid.org/0000-0003-4982-4441

Bo Hyun Kim https://orcid.org/0000-0001-9632-9457

\section{REFERENCES}

1. Lim H, Devesa SS, Sosa JA, Check D, Kitahara CM. Trends in thyroid cancer incidence and mortality in the United States, 1974-2013. JAMA 2017;317:1338-48.

2. Roman S, Lin R, Sosa JA. Prognosis of medullary thyroid carcinoma: demographic, clinical, and pathologic predictors of survival in 1252 cases. Cancer 2006;107:2134-42.

3. Droz JP, Rougier P, Goddefroy V, Schlumberger M, Gardet P, Parmentier C. Chemotherapy for medullary cancer of the thyroid. Phase II trials with adriamycin and cis-platinum administered as monochemotherapy. Bull Cancer 1984;71:1959.

4. Schlumberger M, Abdelmoumene N, Delisle MJ, Couette JE. Treatment of advanced medullary thyroid cancer with an alternating combination of $5 \mathrm{FU}$-streptozocin and $5 \mathrm{FU}$-dacarbazine. The Groupe d'Etude des Tumeurs a Calcitonine (GETC). Br J Cancer 1995;71:363-5.

5. Schwartz DL, Rana V, Shaw S, Yazbeck C, Ang KK, Morrison $\mathrm{WH}$, et al. Postoperative radiotherapy for advanced medullary thyroid cancer: local disease control in the modern era. Head Neck 2008;30:883-8.
6. Valerio L, Pieruzzi L, Giani C, Agate L, Bottici V, Lorusso L, et al. Targeted therapy in thyroid cancer: state of the art. Clin Oncol (R Coll Radiol) 2017;29:316-24.

7. Wells SA Jr, Robinson BG, Gagel RF, Dralle H, Fagin JA, Santoro M, et al. Vandetanib in patients with locally advanced or metastatic medullary thyroid cancer: a randomized, double-blind phase III trial. J Clin Oncol 2012;30:13441.

8. Chougnet CN, Borget I, Leboulleux S, de la Fouchardiere C, Bonichon F, Criniere L, et al. Vandetanib for the treatment of advanced medullary thyroid cancer outside a clinical trial: results from a French cohort. Thyroid 2015;25:386-91.

9. Therasse P, Arbuck SG, Eisenhauer EA, Wanders J, Kaplan RS, Rubinstein L, et al. New guidelines to evaluate the response to treatment in solid tumors. European Organization for Research and Treatment of Cancer, National Cancer Institute of the United States, National Cancer Institute of Canada. J Natl Cancer Inst 2000;92:205-16.

10. Wells SA Jr, Gosnell JE, Gagel RF, Moley J, Pfister D, Sosa JA, et al. Vandetanib for the treatment of patients with locally advanced or metastatic hereditary medullary thyroid cancer. J Clin Oncol 2010;28:767-72.

11. Wedge SR, Ogilvie DJ, Dukes M, Kendrew J, Chester R, Jackson JA, et al. ZD6474 inhibits vascular endothelial growth factor signaling, angiogenesis, and tumor growth following oral administration. Cancer Res 2002;62:4645-55.

12. Robinson BG, Paz-Ares L, Krebs A, Vasselli J, Haddad R. Vandetanib $(100 \mathrm{mg})$ in patients with locally advanced or metastatic hereditary medullary thyroid cancer. J Clin Endocrinol Metab 2010;95:2664-71.

13. Massicotte MH, Brassard M, Claude-Desroches M, Borget I, Bonichon F, Giraudet AL, et al. Tyrosine kinase inhibitor treatments in patients with metastatic thyroid carcinomas: a retrospective study of the TUTHYREF network. Eur J Endocrinol 2014;170:575-82.

14. Werner RA, Schmid JS, Muegge DO, Luckerath K, Higuchi $\mathrm{T}$, Hanscheid $\mathrm{H}$, et al. Prognostic value of serum tumor markers in medullary thyroid cancer patients undergoing vandetanib treatment. Medicine (Baltimore) 2015;94:e2016.

15. Tiedje V, Ting S, Walter RF, Herold T, Worm K, Badziong J, et al. Prognostic markers and response to vandetanib therapy in sporadic medullary thyroid cancer patients. Eur J Endocrinol 2016;175:173-80.

16. Werner RA, Schmid JS, Higuchi T, Javadi MS, Rowe SP, Markl B, et al. Predictive value of $18 \mathrm{~F}-\mathrm{FDG}$ PET in patients with advanced medullary thyroid carcinoma treated with 
vandetanib. J Nucl Med 2018;59:756-61.

17. Valerio L, Bottici V, Matrone A, Piaggi P, Viola D, Cappagli $\mathrm{V}$, et al. Medullary thyroid cancer treated with vandetanib: predictors of a longer and durable response. Endocr Relat Cancer 2020;27:97-110.

18. Subbiah V, Velcheti V, Tuch BB, Ebata K, Busaidy NL, Cabanillas ME, et al. Selective RET kinase inhibition for patients with RET-altered cancers. Ann Oncol 2018;29:186976.

19. Perez-Soler R, Saltz L. Cutaneous adverse effects with HER1/EGFR-targeted agents: is there a silver lining? J Clin Oncol 2005;23:5235-46.

20. Morabito A, Piccirillo MC, Costanzo R, Sandomenico C, Carillio G, Daniele G, et al. Vandetanib: an overview of its clinical development in NSCLC and other tumors. Drugs
Today (Barc) 2010;46:683-98.

21. Rosen AC, Wu S, Damse A, Sherman E, Lacouture ME. Risk of rash in cancer patients treated with vandetanib: systematic review and meta-analysis. J Clin Endocrinol Metab 2012;97:1125-33.

22. Trimboli P, Castellana M, Virili C, Giorgino F, Giovanella L. Efficacy of vandetanib in treating locally advanced or metastatic medullary thyroid carcinoma according to RECIST criteria: a systematic review and meta-analysis. Front Endocrinol (Lausanne) 2018;9:224.

23. Resteghini C, Cavalieri S, Galbiati D, Granata R, Alfieri S, Bergamini C, et al. Management of tyrosine kinase inhibitors (TKI) side effects in differentiated and medullary thyroid cancer patients. Best Pract Res Clin Endocrinol Metab 2017;31:349-61. 